\title{
Optical role of randomness for structured surfaces
}

\author{
Johansen, Villads Egede
}

Published in:

Applied Optics

Publication date:

2014

Document Version

Publisher's PDF, also known as Version of record

Link back to DTU Orbit

Citation (APA):

Johansen, V. E. (2014). Optical role of randomness for structured surfaces. Applied Optics, 53(11), 2405-2415.

\section{General rights}

Copyright and moral rights for the publications made accessible in the public portal are retained by the authors and/or other copyright owners and it is a condition of accessing publications that users recognise and abide by the legal requirements associated with these rights.

- Users may download and print one copy of any publication from the public portal for the purpose of private study or research.

- You may not further distribute the material or use it for any profit-making activity or commercial gain

- You may freely distribute the URL identifying the publication in the public portal

If you believe that this document breaches copyright please contact us providing details, and we will remove access to the work immediately and investigate your claim 


\title{
Optical role of randomness for structured surfaces
}

\author{
Villads Egede Johansen \\ Technical University of Denmark, Department of Mechanical Engineering, 2800 Kgs. Lyngby, Denmark (vejo@mek.dtu.dk)
}

Received 2 January 2014; revised 2 January 2014; accepted 26 February 2014; posted 5 March 2014 (Doc. ID 198272); published 7 April 2014

\begin{abstract}
It has long been known that random height variations of a repeated nanoscale structure can give rise to smooth angular color variations instead of the well-known diffraction pattern experienced if no randomization is present. However, until now there have been few publications trying to explain this and similar phenomena taking outset in electromagnetic theory. This paper presents a method for analyzing farfield reflection from a surface constructed by translated instances of a given structure. Several examples of the effect of random translations are given. (c) 2014 Optical Society of America

OCIS codes: $\quad$ (030.1670) Coherent optical effects; (050.1960) Diffraction theory; (240.6700) Surfaces; (290.0290) Scattering; (330.1690) Color; (330.7326) Visual optics, modeling.

http://dx.doi.org/10.1364/AO.53.002405
\end{abstract}

\section{Introduction}

In the 17th century, Robert Hooke discovered how dielectric structures with size features comparable to the wavelengths of light were an important part of the color appearance of certain animals he studied under a microscope [1]. However, the theory of light was not well developed at the time; there were no computers, and the optical microscopes simple, so the field of structural colors remained rather untouched. In recent decades, the invention of electron microscopes, the computer, and the wave theory of light in the last century have made it possible to do more in-depth investigation of this field that has more than 500 million years of history in nature [2].

Controlling light reflection by interaction with structures is crucial for many applications. Retroreflectors, aluminum foil, solar cells, and security holograms are just a few examples encountered frequently in everyday life. These examples also show that there are many motivations for controlling color appearance of an object besides the visual appearance, and that improving the understanding of light's interaction with surfaces can improve a wide range of engineering applications and possibly initiate new inventions.

$1559-128 \mathrm{X} / 14 / 112405-11 \$ 15.00 / 0$

(C) 2014 Optical Society of America
Many important contributions to the understanding of structural colors have been uncovered by studying the nanostructure of the wing of the Morpho rhetenor butterfly. The results range from what is presented in [3] and up until present day, where it is possible to make a $3 \mathrm{D}$ rendered model of the wing's color appearance and reflection based on modeling of the structure of the wing [4]. On the Morpho type in general, excellent works have been published dating longer back, see, e.g., [뭏]. The reasons for why this structure especially has become so central in the analysis of structural colors are probably (1) the fact that its structure is more or less invariant along one axis, leaving it possible to simulate only a cross section of the model; (2) the rather simple shape of the structure, making it possible at an early stage to obtain good results just by analyzing it as a multilayer structure and then extend the model more and more to refine the results.

One of the properties that still needs investigation is how to model random displacements of the individual, repetitive structures present in the Morpho rhetenor's wing (see Fig. 3.45 in [7] and the following description in [8]). By numerical as well as practical experiments, it has been shown how these random displacements of a periodic structure seem to smooth out the otherwise strong diffractive effects, which are expected from reflections of periodic structures. A method for incorporating randomness without doing 
simulations using a large numerical domain is still missing. In this paper, we will present a method for analyzing repeated structures with (or without) random translations of the elements. As a benchmark, previously published results on the random behavior related to the Morpho rhetenor butterfly will be used, but the method has a much broader aim than this: it should make it possible to design structures with new color effects, and it should help in giving a better intuitive understanding of the influence of different kinds of randomization of structures (e.g., in-plane versus height displacements). This paper focuses on visible light, but the method is applicable in all parts of the electromagnetic spectrum.

The rest of the paper is organized as follows. Section 2 motivates the work in this paper by showing examples in the literature where it can be used. Section 3 presents the background needed to analyze the random effects. Section $\underline{4}$ applies the knowledge to results obtained in earlier studies and shows how these could have been predicted using the presented method. Section 5 gives some examples on what influence the randomness would have on the color appearance of a surface for some specific cases, and finally Section $\underline{6}$ concludes on the presented results.

\section{Observations of Randomness in the Literature}

In this paper, we will focus on surfaces comprised of repeated unit structures in the $x, y$ plane with some per unit height displacement in the $z$ direction (see Fig. 1 for an example of a repeated structure). Structures fitting to this description are found in many places in the literature of optics. Partly in cases where the translations of the copied structures are deterministic and partly where the description contains random parameters. To motivate the present paper, some publications relying on one unit structure repeated throughout a domain are listed below:

- Several publications exist considering the influence of randomness of binary gratings: in [9] an experimental study of in-plane randomness is conducted with results that can also be explained from

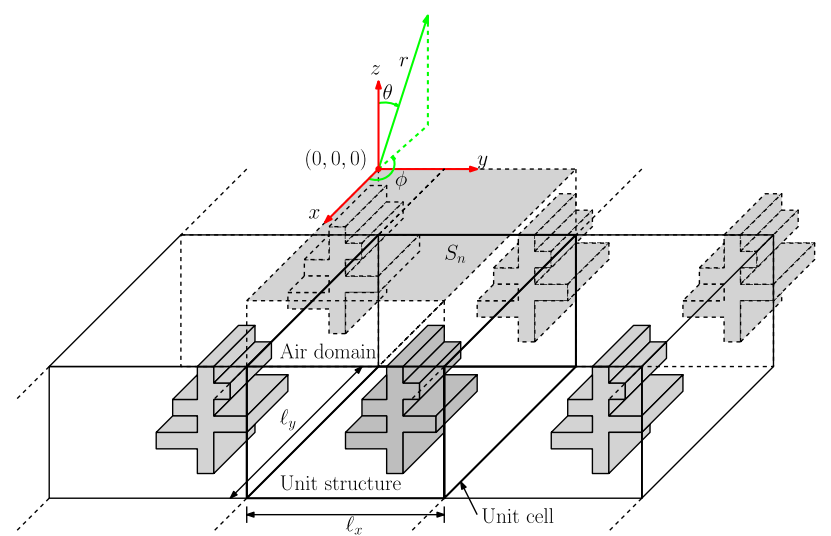

Fig. 1. Same structure divided into cells with an air domain above. the present paper; and in [10] some binary height variations of gratings are studied that also can be analyzed using the present paper.

- Phase gratings using the properties of randomness for concrete products such as, e.g., a surface giving controlled angular redirection of light for windows to improve indoor lighting environment has been presented in [11]. Here the effect of in-plane randomness is observed-but not explained.

- Designing random disorder is also seen in photovoltaic solar cell applications as presented in, e.g., [12]. In [12], the method presented in the present paper could have been used to investigate the effect of different randomization parameters before utilizing full wave simulations for a detailed study.

For testing the usefulness of the presented method, studies of the nanostructure of the Morpho butterfly's wing will be used, since it has undergone many studies in the last decades with focus on different aspects of randomness. The works to be used are:

- "Detailed electromagnetic simulation for the structural color of butterfly wings [13]," which shows numerically how the far-field response of one lit Morpho butterfly ridge (which is the "unit structure" of this butterfly's wing) almost corresponds to the response of many random height translated elemenents;

- "Reproduction, mass-production, and control of the Morpho-butterfly's blue [14]," which shows by experiment how a binary random pattern with a structure on top can be used to generate a smooth color effect compared to no randomization;

- "Numerical analysis on the optical role of nanorandomness on the Morpho butterfly's scale [15]," which conducts several numerical experiments with different kinds of randomization parameters to investigate the effect of these, and;

- "Detailed simulation of structural color generation inspired by the Morpho butterfly [16]," whichamong other numerical experiments-contains an analysis of the effect of different maximum heights chosen for randomization.

The method to be presented will be confirmed by these observations.

\section{Method for Analyzing Random Translations}

In this section, a method for analyzing (random) translations of structures is presented. It is described for a 3D general case, even though the examples later on will be $2 \mathrm{D}$. The analysis is performed for time harmonic waves using the time factor $e^{j \omega t}$.

\section{A. Huygens' Principle}

Consider an electromagnetic structure with its volumes divided into different cells-which in this article will be referred to as units when all cells contain the same structure-as seen in Fig. 1. By Huygens' principle and the image principle [17]], it is possible to calculate the (near-field as well as) far-field contribution in the upper hemisphere from 
any structure by considering the so-called equivalent surface currents, $\mathbf{J}_{e}$, calculated on an infinite plane above the structure. An example of this could be the structure seen in Fig. 1 if the plane $S_{n}$ is extended to infinity. Due to the linearity of Maxwell's equations, the plane can be split into parts with each of their far-field contribution, and by summing them we still arrive at the same total far-field contribution.

Now by defining a plane above the structure in Fig. 1 and splitting it into parts such that each part follows the projection of the cell on the plane-like $S_{n}$ in Fig. 1-the magnetic far-field contribution for the $\mathbf{H}$-field, $\mathbf{H}^{f}$, can be found as (see, e.g., Chapter 6 in [17])

$$
\mathbf{H}^{f}(\theta, \phi)=\sum_{n \in \mathbb{N}} \mathbf{H}_{n}^{f}(\theta, \phi),
$$

where

$$
\mathbf{H}_{n}^{f}(\theta, \phi)=-j k \frac{e^{-j k r}}{4 \pi r} \hat{\mathbf{r}} \times \int_{S_{n}} \mathbf{J}_{e} e^{j k \hat{\mathbf{r}}(\theta, \phi) \cdot \mathbf{r}_{o}} \mathrm{~d} S_{n},
$$

where $k=2 \pi / \lambda$ is the wavenumber, the $S_{n}$ 's $(n \in \mathbb{N})$ are all unique parts making up the total surface $S, r$ is the distance from an arbitrarily located origin on $S_{n}$ to the evaluation point (since $r$ is used in places where phase information is not important, it is assumed constant), $\hat{\mathbf{r}}=(\sin \theta \cos \phi, \sin \theta \sin \phi, \cos \theta)$ is the direction toward the far-field evaluation point, and $\mathbf{r}_{0}=(x, y, z) \in S_{n}$ is the position vector (as measured from the origin) to a point on $S_{n}$. The variables $x, y, z, \theta, \phi$ are all defined as in Fig. 1 .

In the far-field and free space, the radiated wave locally approaches a TEM (transverse electromagnetic) wave, and one can therefore make use of the relation between the electric and magnetic field due to this behavior:

$$
\mathbf{E}^{f}=\eta \mathbf{H}^{f} \times \hat{\mathbf{r}},
$$

where $\eta \approx 377 \Omega$ is the free-space impedance, to calculate the irradiance $E$-which equals the magnitude of Poynting's vector-as

$$
E=\frac{1}{2}\left|\mathbf{E}^{f} \times \overline{\mathbf{H}^{f}}\right|=\frac{1}{2} \eta\left|\mathbf{H}^{f}\right|^{2}=\frac{1}{2} \eta\left|\sum_{n \in \mathbb{N}} \mathbf{H}_{n}^{f}\right|^{2},
$$

which will be used later on in the analysis.

\section{B. Translation of Lit Structures}

We want to manipulate Eq. (4) such that it includes (random) height/length translations of structures in all three spatial directions, $\Delta \mathbf{r}=(\Delta x, \Delta y, \Delta z)$ (see Fig. 2 for a planar example). Considering one of these structures, it is lit by a plane wave with propagation direction $\hat{\mathbf{k}}$ as also indicated in the figure. This means, first of all, that by translating it, we introduce a phase lag, $\Delta p$, on the phase that the incoming wave meets the structure with, which can be described as

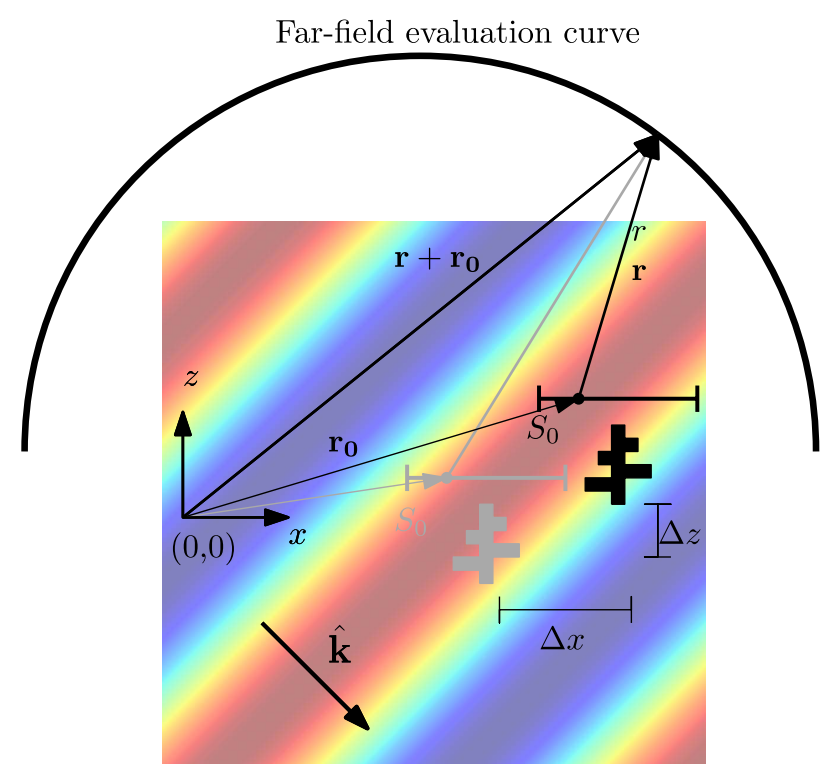

Fig. 2. Geometry of the translation of a structure lit by a plane electromagnetic wave.

$$
\Delta p=-\hat{\mathbf{k}} \cdot \Delta \mathbf{r} / \lambda
$$

In relation to the evaluation of the far field, there is also a difference, since the vector $\mathbf{r}_{0}$ has changed so that it now is represented by

$$
\mathbf{r}_{0}^{\text {new }}=\mathbf{r}_{0}+\Delta \mathbf{r},
$$

and this influences the far-field transformation in Eq. (2). We do not need to update the distance, $r$, since the change in contribution to the distance is negligible, which is in agreement with the assumptions made in the derivation of far-field radiation in the first place [17].

Modifying Eq. (2) then gives

$$
\begin{aligned}
\mathbf{H}_{n}^{f, t}(\theta, \phi)= & -e^{-j 2 \pi \hat{\mathbf{k}} \cdot \Delta \mathbf{r} / \lambda} j k \frac{e^{-j k r}}{4 \pi r} \hat{\mathbf{r}} \\
& \times \int_{S_{n}} \mathbf{J}_{e} e^{j k \hat{\mathbf{r}}(\theta, \phi) \cdot\left(\mathbf{r}_{o}+\Delta \mathbf{r}\right)} \mathrm{d} S_{n} \\
= & -e^{-j k(\hat{\mathbf{k}} \cdot \Delta \mathbf{r}-\hat{\mathbf{r}}(\theta, \phi) \cdot \Delta \mathbf{r})} j k \frac{e^{-j k r}}{4 \pi r} \hat{\mathbf{r}} \\
& \times \int_{S_{n}} \mathbf{J}_{e} e^{j k \hat{\mathbf{r}}(\theta, \phi) \cdot \mathbf{r}_{o}} \mathrm{~d} S_{n} \\
= & e^{-j k(\hat{\mathbf{k}}-\hat{\mathbf{r}}(\theta, \phi)) \cdot \Delta \mathbf{r}} \mathbf{H}_{n}^{f}(\theta, \phi),
\end{aligned}
$$

where the superscript $t$ indicates that it is the translated response, and $\mathbf{J}_{e}$ is still the equivalent surface current of the untranslated structure. This result simply describes the angular-dependent change in phase to the contribution that is seen in an observation point when moving the structure around in a lit domain. 


\section{Irradiance of Translated Structures}

In this paper, we will focus on one basic structure that will exist in several translated instances in the domain (that is, the structure itself will not be pertubed). This structure will be referred to as the unit structure. Due to superposition, there can in principle be several unit structures as long as they do not overlap.

If we assume that we can find the far field radiance of this structure - or at least an adequate approximation of that-we can then sum over this response with the correct translations to find how a system of these structures will reflect light. The validity of the assumption will be discussed in Section 3.G. The far-field radiance has for similar type of problems been calculated by transforming the equivalent surface current simulated over a unit structure using either periodic boundary conditions [18] or radiation boundary conditions [4] depending on the setup.

By having the far-field response for one unit structure, $\mathbf{H}_{0}^{f}$, we can then find the total far-field irradiance as

$$
\begin{aligned}
E & =\frac{1}{2} \eta\left|\sum_{n} e^{-j k(\hat{\mathbf{k}}-\hat{\mathbf{r}}) \cdot \Delta \mathbf{r}_{n}} \mathbf{H}_{0}^{f}\right|^{2} \\
& =\frac{1}{2} \eta\left|\mathbf{H}_{0}^{f}\right|^{2} \underbrace{\left.\sum_{n} e^{-j k(\hat{\mathbf{k}}-\hat{\mathbf{r}}) \cdot \Delta \mathbf{r}_{n}}\right|^{2}}_{=\mathrm{AF}} .
\end{aligned}
$$

In this formulation, the response of the unit structure is isolated such that the total irradiance is just the response of the unit structure multiplied with the magnitude of some function squared. This function we denote the array factor (AF), since it plays the same role as an AF does in antenna theory [17]. Note how the exponent inside the $\mathrm{AF}$ has an angulardependent term, $\hat{\mathbf{r}}(\phi, \theta)$, and a term dependent on the direction of the incoming wave, $\hat{\mathbf{k}}$.

A similar expression appears in [19], but is presented on the basis of scalar diffraction theory.

\section{Radiance of Translated Structures}

The response of the eye is proportional to radiance and not irradiance [20], meaning that for appearance and color purposes, we need to convert irradiance to radiance.

From [21] it is shown that the irradiance only having a component normal to the observation surface (which is true for a detector in the far field) is related to the radiant intensity, $I$, by

$$
I(\theta, \phi)=r^{2} E(\theta, \phi),
$$

and also from [21] the relation between radiant intensity and radiance, $L$, is given as

$$
L(\theta, \phi)=\frac{I(\theta, \phi)}{A_{s} \cos \theta}=\frac{r^{2}}{A_{s} \cos \theta} E(\theta, \phi),
$$

where $A_{s}$ is the area of the surface of the lit structure. This means we can write the total radiance for a lit surface with repeated structures as

$$
\begin{aligned}
L(\theta, \phi) & =\frac{r^{2} \eta}{2 A_{s} \cos \theta}\left|\mathbf{H}_{0}^{f}(\theta, \phi)\right|^{2}\left|\sum_{n} e^{-j k(\hat{\mathbf{k}}-\hat{\mathbf{r}}(\theta, \phi)) \cdot \Delta \mathbf{r}_{n}}\right|^{2} \\
& =\underbrace{\frac{r^{2} \eta}{2 A_{0} \cos \theta}\left|\mathbf{H}_{0}^{f}(\theta, \phi)\right|^{2}}_{=\text {unit response }} N \underbrace{\left|\frac{1}{N} \sum_{n} e^{-j k(\hat{\mathbf{k}}-\hat{\mathbf{r}}(\theta, \phi)) \cdot \Delta \mathbf{r}_{n}}\right|^{2},}_{=\mathrm{SAF}}
\end{aligned}
$$

where $A_{0}$ is the surface of $\mathbf{H}_{0}^{f}$ and $N$ is the number of summations in the sum, which means that $A_{s}=N \cdot A_{0}$. The peculiarity of having $N$ present twice is to scale the last product such that it peaks at unity. Since the AF is now scaled by the number of units, we will refer to it as the scaled array factor (SAF).

The expression in Eq. (11) will be the foundation for all following analyses, as it shows how a prediction of the reflection from the unit structure (this we denote unit response) and a knowledge of the position of its instances (the $\Delta \mathbf{r}_{n}{ }^{\prime}$ s) can give a complete description of the reflected radiance. Furthermore it decouples the positioning of the structures from the response of the structure, making it possible to analyze each part individually.

\section{E. 1D Version of Formulas}

In the rest of this paper, we will consider structures only with variation in the $x$ and $z$ directions and traveling in the $x, z$ plane to keep the examples simple. This means that the results will be invariant in the $y$ plane, and we therefore put $\phi=90^{\circ}$ [22]. To indicate this, the following notation will be applied in the rest of this paper:

$$
\begin{aligned}
L(\theta)= & \frac{r \eta}{2 d_{0} \cos \theta}\left|\mathbf{H}_{0}^{f}\left(\theta, \phi=90^{\circ}\right)\right|^{2} N \\
& \times\left|\frac{1}{N} \sum_{n} e^{-j k\left(\hat{\mathbf{k}}-\hat{\mathbf{r}}\left(\theta, \phi=90^{\circ}\right)\right) \cdot \Delta \mathbf{r}_{n}}\right|^{2},
\end{aligned}
$$

where $d_{0}$ is the length of the unit structure instead of the area. The validity of the SAF concept has been tested using this version of the formula in Appendix A.

\section{F. Interpretation of Array Factor}

From Eqs. (11) and (12) it is seen how the total radiance is the product of a unit radiance and the absolute squared SAF. This means that they can be treated independently of each other, and in this paper we will focus only on the SAF and not consider a specific unit response, but instead pose the problem: for a certain unit structure, what role does different randomizations play? The effect of different choices 


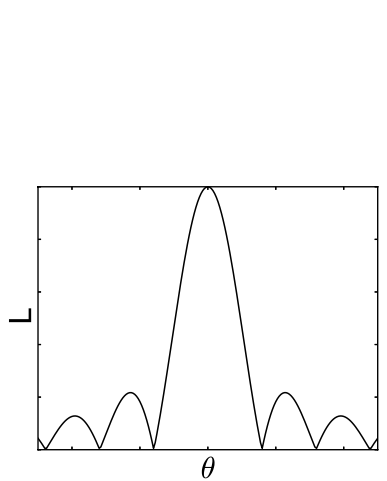

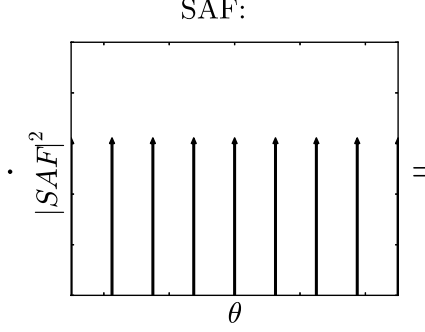

(b)

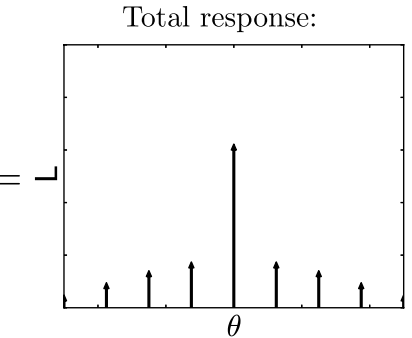

(c)

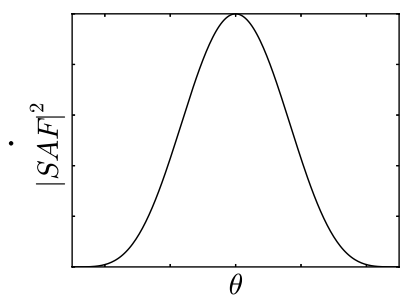

(d)

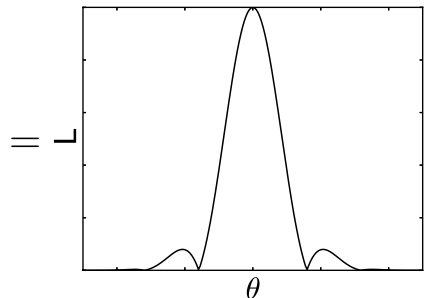

(e)

Fig. 3. Example of how a structure with some unit response (a) is influenced by different SAFs. In (b) the SAF for a strictly periodic structure is shown (the comb function), and the effect of combining the structure giving the unit response in (a) with the pattern giving rise to the SAF in (b) is shown in (c). The arrows indicate that all energy is emitted at discrete points. If the SAF is as it is in (d), then the response will end up as seen in (e).

of translations (that is, different SAFs) of the same structure and its influence is exemplified in Fig. 3 .

\section{G. Limitations Due to Assumptions}

In Sections 3.C and 3.D, we have assumed that all structures on a surface have the same response (except for the translation part), and it is important to clarify when and to what extent these assumptions hold. This is discussed in the following and in Appendix A where a numerical example is presented.

\section{Dependence of Surrounding Geometry}

If a unit structure is simulated sitting in a periodic structure as in Fig. 4(a), and then in reality is sitting in some random structure as in Fig. 4(b), it is clear that the actual far-field response is somewhat different, since the electromagnetic coupling to the neighboring elements has changed. It is therefore necessary that the resulting change in far-field response is small or averages out over many elements, and it is also necessary that the displaced unit structures do not shadow each other. This will in general become less important for large unit structures, since the interelemental coupling in most cases then will be negligible.

It should also be noted that all practical structures have finite sizes, and the unit structures at the edges probably will have another far-field response due to difference in the surrounding geometry. If the surface is large compared to the area occupied by the outer unit structures, this effect should be negligible.

\section{Equivalent Surface Assumption}

We have assumed that there exists a plane surface, $S_{0}$, above the unit structure on which we can calculate the equivalent surface current, $\mathbf{J}_{e}$, and then find the needed unit response from here. Furthermore, we assume that we can calculate the total response from some configuration of unit structures by stitching a plane surface, $S$, together by these surface currents with a first-order phase-correction term taking their translation into account.

For the above assumptions to work well, we recommend putting the far-field transformation surface as close to the electromagnetic structure as possible for two reasons: the first is to take as much energy as possible into account and thereby catching the behavior in the best way possible; the second is that it will minimize unwanted contributions from the surroundings through the surface (e.g., if there are periodic boundary conditions).

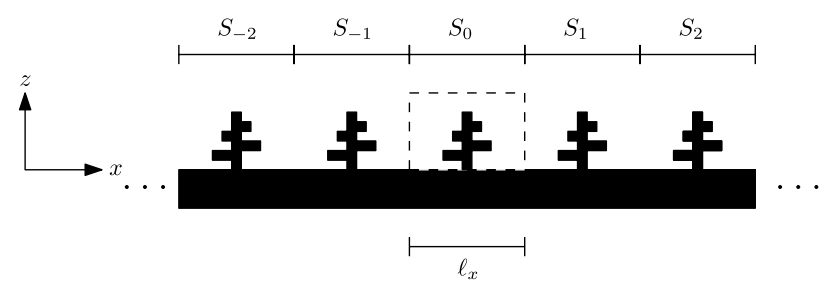

(a)

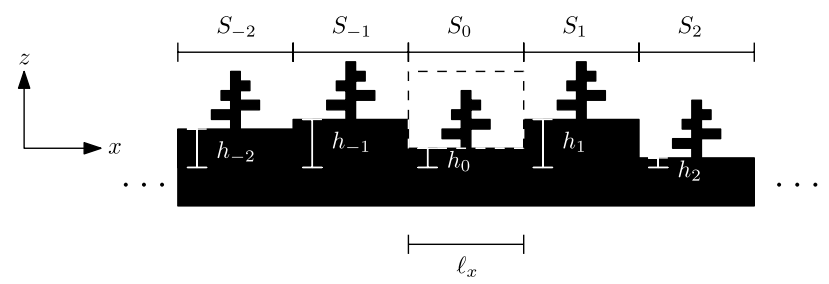

(b)

Fig. 4. (a) Strictly periodic structure. (b) The same structure but with per period height translations. 


\section{Analysis of Random Structures}

This section will apply Eq. (12) to different cases-all focusing on explaining the influence of (random) translations of well-defined structures as observed by experiments and simulations in the literature. These examples are chosen to show how randomness can be analyzed and to provide understanding of the effects due to translational randomization.

All results will be in 2D and furthermore with an incoming wave perpendicular to the surface. This means that some important features such as the dependence on the direction of the incoming wave, $\hat{\mathbf{k}}$, will not be discussed in this paper.

\section{A. No Randomness: A Special Case Called Diffraction}

To introduce the concept and confirm its validity for a known (but trivial) case, the first example will be the rather simple case of a repeated structure with no randomization.

Consider an infinitely periodically repeated unit structure within a domain [see Fig. 4(a)]. If this structure is lit with a normal incident plane wave, then we have that

$$
\hat{\mathbf{k}}=(0,0,-1), \quad \Delta \mathbf{r}_{n}=\left(n \ell_{x}, 0,0\right), \quad n \in \mathbb{Z},
$$

where $\ell_{x}$ is the period with which the structure is translated. The SAF for this structure then becomes

$$
\operatorname{SAF}(\theta)=\frac{1}{N} \sum_{n \in \mathbb{Z}} e^{-j k(\hat{\mathbf{k}}-\mathbf{r}(\theta)) \cdot \Delta \mathbf{r}_{n}}=\frac{1}{N} \sum_{n \in \mathbb{Z}} e^{j k n \ell_{x} \sin \theta} .
$$

For infinite repetition, the limit of the summation inside the norm can be expressed as [23]

$$
\sum_{n \in \mathbb{Z}} e^{j 2 \pi n \frac{\ell_{x}}{\lambda} \sin \theta}=\sum_{n \in Z} \delta\left(\frac{\ell_{x}}{\lambda} \sin \theta-n\right)=: \operatorname{comb}\left(\frac{\ell_{x}}{\lambda} \sin \theta\right),
$$

where $\delta$ is the Dirac delta function, and the name $c o m b$ is given due to the function's resemblance with a comb [see Fig. 3(b)]. It is also referred to as the shah function. Since $\delta(x)=0, \quad \forall x \in \mathbb{R} \backslash\{0\}$, reflection from this structure will only appear when

$$
\frac{\ell_{x}}{\lambda} \sin \theta=m \in \mathbb{Z}
$$

and have strong intensity. The relation in Eq. (16) is called the grating equation and $m$ is normally referred to as the mode number. It is a well-known result, explaining, e.g., the rainbow effect seen when observing a compact disc (CD), which consists of equally spaced grooves used to store the data. This behavior is referred to as diffraction and is seen to be explained by Eq. (12) as expected.

\section{B. Random Height Variation of Repeated Structure}

Consider the same setup as before with an infinitely repeated structure [see Fig. $\underline{4(\mathrm{a})]}$. In the analysis of the coloration of the Morpho butterfly, numerical simulations in [13] shows that by adding a random height variation to each unit, the total response will start to resemble the unit response with overlayed high-frequency ripples. To analyze this, we apply a random height translation to each unit drawn from a uniform distribution with values between 0 and $\lambda_{\max }$, where $\lambda_{\max }$ is the longest wavelength in the analysis. The final structure will then look like the one seen in Fig. 4(b). The incoming wave is still normal to the surface, so the components for the SAF of this system are

$$
\hat{\mathbf{k}}=(0,0,-1), \quad \Delta \mathbf{r}_{n}=\left(n \ell_{x}, 0, \ell_{z, n}\right), \quad n \in \mathbb{Z},
$$

where $\ell_{x}$ is the period and $\ell_{z, 0}, \ell_{z, 1}, \ell_{z,-1}, \ell_{z, 2} \ldots$ is a sequence of numbers drawn from a uniform distribution with values between 0 and $\lambda_{\max }$. This means that the SAF now takes the form

$$
\begin{aligned}
\operatorname{SAF}(\theta) & =\frac{1}{N} \sum_{n \in \mathbb{Z}} e^{-j k\left(n \ell_{x} \sin \theta-(\cos \theta+1) \ell_{z, n}\right)} \\
& =\frac{1}{N} \sum_{n \in \mathbb{Z}} e^{-j k n \ell_{x} \sin \theta} e^{j k(\cos \theta+1) \ell_{z, n},}
\end{aligned}
$$

where we see that the first product in the summation comes from the periodic translation, and the second from the random height translation. The minus sign in the second product indicates that positive height displacements reflect the phase earlier, and the angle dependent $1+\cos \theta$ can be interpreted geometrically as the extra added distance the wave has to travel. That is, for specular reflection where $\theta=0$, the wave will also travel the same phase less than it did when reaching the structure, but for other values of $\theta$, it will travel a bit longer, and therefore not as much negative phase lag will be removed.

A typical response of Eq. (18) is seen in Fig. 5(a) for a finite number of structures $(N=100)$. It is seen how $\ell_{z, n}$ should vary between zero and half a wavelength to get a SAF where no diffraction pattern is present and also that if there is no randomization, then the SAF has sharp intensities peaking in the grating modes that can be calculated from Eq. (16). The finite number of repetitions spreads out the intensities from just a single angle to a small angular area. It is also seen that there is a gradual change from pure diffraction to no diffraction with the change of wavelength. For larger maximum values of $\ell_{z, n}$, the diffraction is in general weakly present if present at all.

A good way of describing the above observations intuitively is by considering what phase is most probable to be observed in a certain far-field direction, and if there is no preferred phase then there is no possibility of interference, whereas if there is a preference of a phase, then that will give rise to interference effects (e.g., if the phase is varying in a smaller interval than $\theta \in\left[0^{\circ}, 360^{\circ}\right]$ ). 

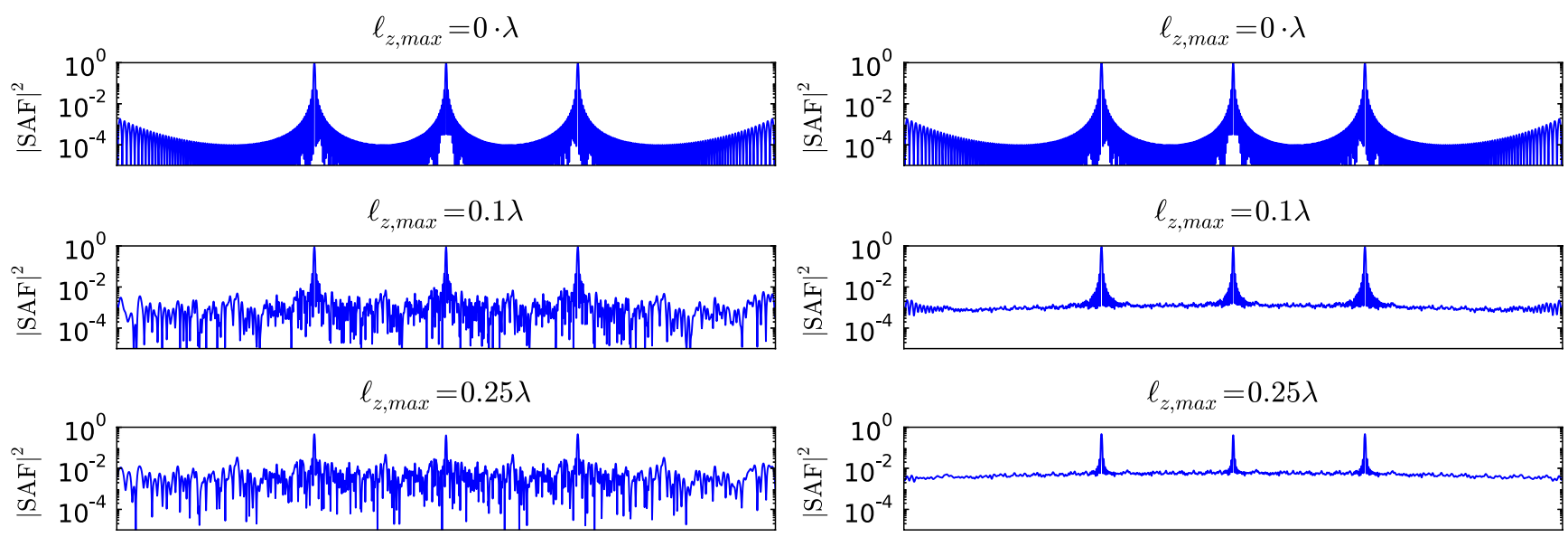

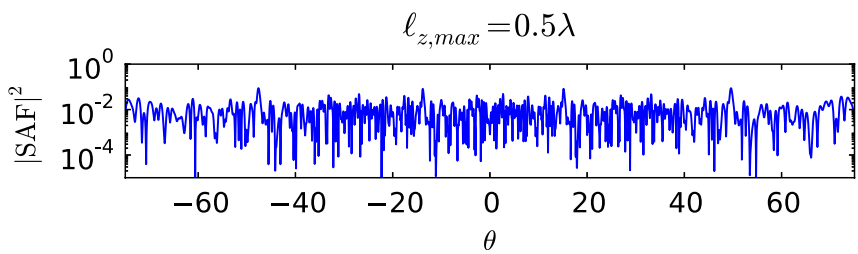

(a)

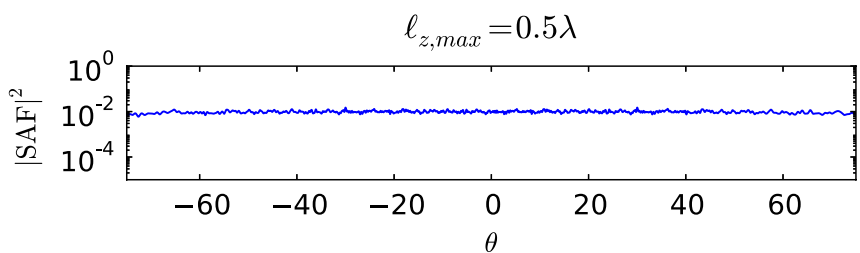

(b)

Fig. 5. (a) Numerical calculation of Eq. (18) with $\ell_{z, n}$ picked uniformly from $\left[0, \ell_{z, \max }\right]$ and $N=100$ repetitions and a periodicity in $x$ of $\ell_{x}=2 \lambda$. By changing $\ell_{x}$ the SAF would either be dilated or constricted such that the grating modes still match Eq. (16). (b) 100 averages of the setup in (a) using Eq. (19).

These results can be confirmed by [15] where they report a clearly visible diffraction pattern for small variation and show that larger variations are needed to minimize the effect. Furthermore it can be seen in Fig. 4(c) in [15] how the $380 \mathrm{~nm}$ still shows the firstorder diffraction at $\theta=\arcsin (380 / 400) \approx 72^{\circ}$, and otherwise a random pattern with strong "noise" and a zero-order mode is still present, since the variations are smaller than a wavelength, and furthermore how longer wavelengths (as just shown) gives a larger contribution to mode 0 (when the relative reflection from Fig. 3 in [15] is taken into account). Furthermore, the same analysis reveals the governing effect of Fig. 6 in [16] in the explanation of the results in [16].

\section{Added Incoherence}

In reality, the reflected spectrum has less ripples than in Fig. 5(a). In $[15,24]$, this has been taken into account by averaging the irradiance of a large ensemble to add the effect of incoherence. The explanation for doing so is that the phases of two uncorrelated waves on average will neither add destructively nor constructively, and it is therefore possible just to sum their powers. That is,

$$
|\operatorname{SAF}(\theta)|^{2}=\left\langle\left|\frac{1}{N} \sum_{n \in \mathbb{Z}} e^{-j k n \ell_{x} \sin \theta} e^{j k(\cos \theta+1) \ell_{z, n}}\right|^{2}\right\rangle,
$$

where $\langle\cdot\rangle$ for this equation indicates that the average will be taken of the SAF for many different seeds of $\ell_{z, n}$. By doing this averaging, we end up with the result in Fig. 5(b), where the "noise ripples" are now much smaller. This is in good agreement with the results from [15], and, for an even higher number of averages, the ripples become even smaller.

\section{Triangular Height Distribution}

In [13], the height displacements are chosen from a triangular distribution. Using Eq. (18), where $\ell_{z, n}$ is now drawn from a triangular distribution ranging from 0 to $\ell_{z \text {,max }}$ with the triangular peak placed in $\ell_{z \text { max }} / 2$, gives the results presented in Fig. $\underline{6}$. This result has more visible diffraction than for the uniform samples. In particular it is seen how they are still present for $\ell_{z \text { max }}=1 / 2 \lambda$. Investigating the $\mathrm{Ph} . \mathrm{D}$. thesis on which the article is based [25] reveals that the interval for the triangular distribution is also double the size of the uniform distribution it is compared with. Doing the analysis with a broader distribution gives the results in Fig. 7, and from here it is seen that the modes actually are better suppressed, which explains the choice of distribution in [13]. It was chosen to plot the incoherent/averaged version here, since it is then easier to compare with Fig. $5(\mathrm{~b})$.

\section{Binary Randomized Heights}

In [14], a blue surface without specular reflections is produced to mimic the behavior of the Morpho butterfly wing. For easy fabrication purposes, the randomization of the unit structure is made up by only two heights; that is, $\ell_{z, n}$ in Eq. (18) can take on two values: 0 and $\ell_{z \text {,max }}$. Repeating the setup from before, but now with this binary randomness, we obtain the results presented in Fig. 8 . Here it is seen how a height difference of a quarter of a wavelength 

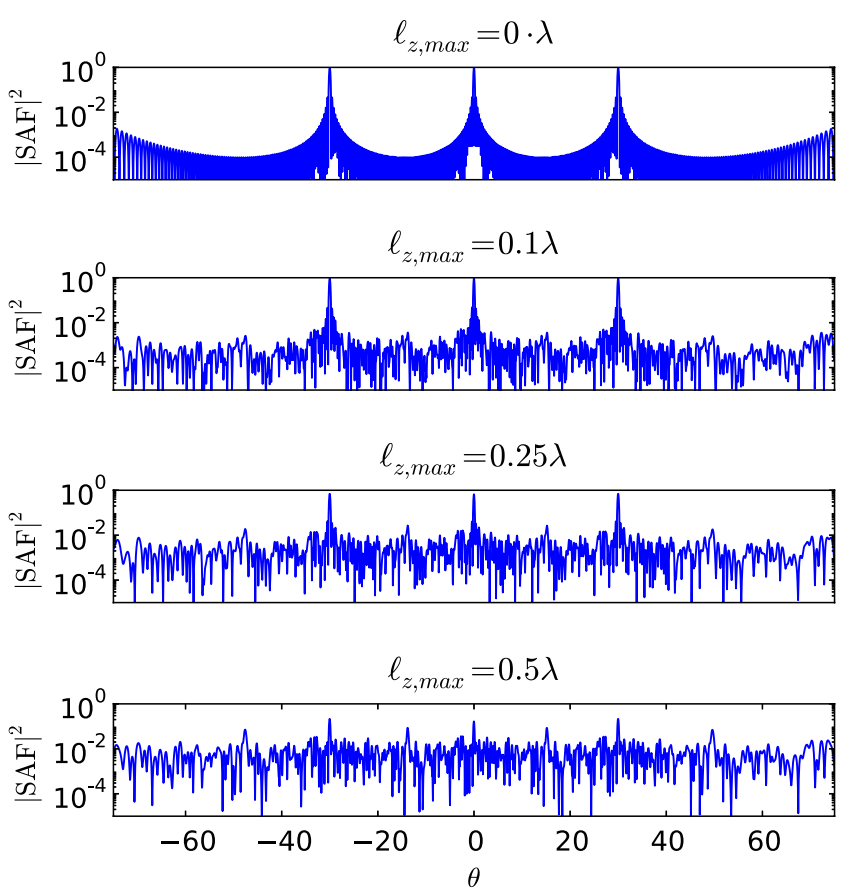

Fig. 6. Results for the same setup as in Fig. 5(a), but now with $\ell_{z, n}$ taken from a triangular distribution.

at the target frequency is needed to get a flat $|\mathrm{SAF}|^{2}$. This is in perfect agreement with the height chosen in [14]. Choosing a height difference on half a wavelength gives strong grating modes. The reason is that for a height difference of $1 / 4 \lambda$, the reflected light is sent back $180^{\circ}$ out of phase and therefore interferes destructively, leaving no preferred directions for the reflected light, whereas if the light travels $1 / 2 \lambda$ extra back and forth it corresponds to a $360^{\circ}$
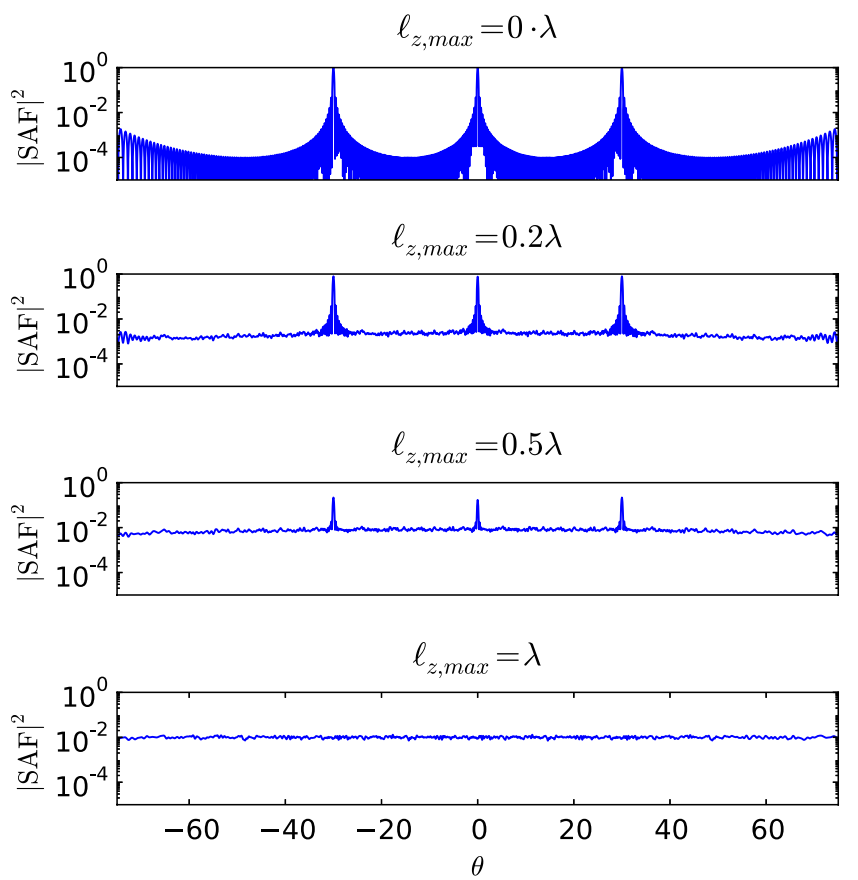

Fig. 7. 100 averages of the same setup as Fig. 6(a), but for double the interval width of the distribution used in Fig. 6(a).
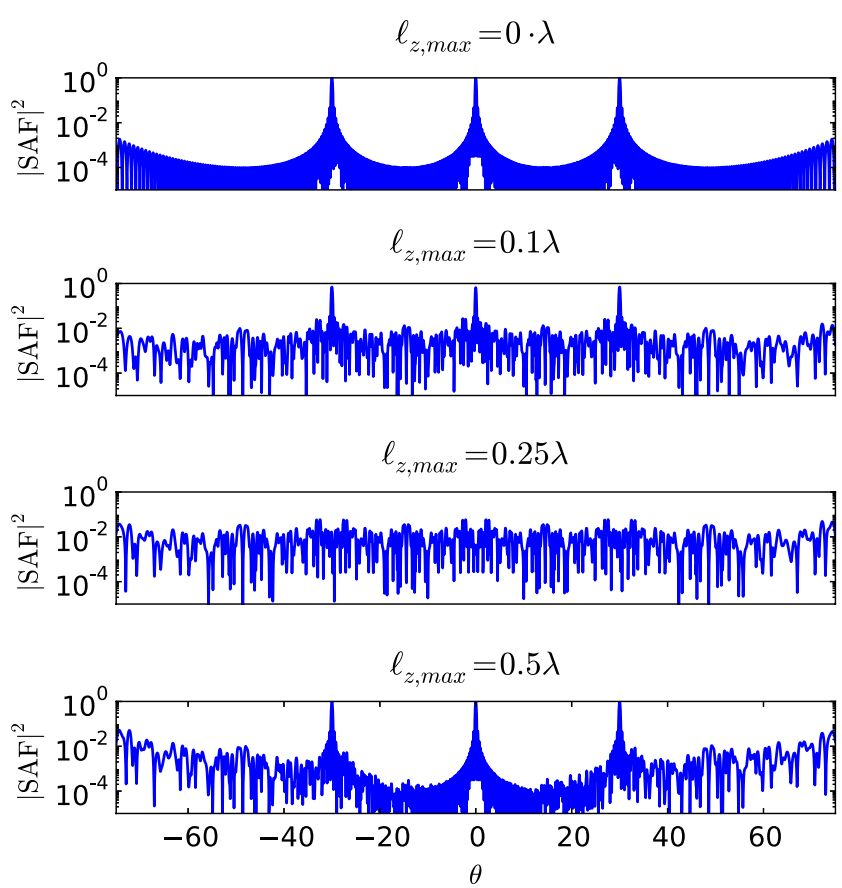

Fig. 8. Results for the same setup as in Fig. $\underline{5(\mathrm{a})}$, but now with $\ell_{z, n}$ only taking the values 0 and $\ell_{z \text {,max }}$.

phase shift, which gives constructive interference and modes as seen in Fig. 5(a). Since the reflected light travels a bit longer than $1 / 2 \lambda$ when reflected back in off-specular directions (taken care of in the equation by the $\cos \theta$ term), the effect of constructive interference wears off more and more for increasing angles, which is also seen on the plot when comparing $\ell_{z, \max }=0$ with $\ell_{z, \max }=1 / 2 \lambda$.

This example shows the need for analyzing specific randomizations, as here $\ell_{z, \max }$ has to be chosen differently, and since the effect only can be expected to work well for a smaller range of wavelengths than in the uniform randomness case. In the design in [14], the wavelengths far from the blue region around $440 \mathrm{~nm}$ are suppressed by the unit structure, thus hiding the diffraction effects that would otherwise have been present.

\section{E. In-Plane Translated Elements}

In [15], in-plane movement is considered; that is, movement in the $x$ direction of a repeated structure. For this setup, the following definitions for the SAF are needed:

$\hat{\mathbf{k}}=(0,0,-1), \quad \Delta \mathbf{r}_{n}=\left(n \ell_{x}+\Delta x_{n}, 0,0\right), \quad n \in \mathbb{Z}$,

where the $\Delta x_{n}$ 's is a sequence of random variables drawn uniformly from the range $\left[-\Delta x_{\max } / 2\right.$, $\left.\Delta x_{\max } / 2\right]$. This gives rise to the following SAF:

$$
\begin{aligned}
\operatorname{SAF}(\theta) & =\frac{1}{N} \sum_{n \in \mathbb{Z}} e^{-j k\left(n \ell_{x}+\Delta x_{n}\right) \sin \theta} \\
& =\frac{1}{N} \sum_{n \in \mathbb{Z}} e^{-j k n \ell_{x} \sin \theta} e^{-j k \Delta x_{n} \sin \theta} .
\end{aligned}
$$



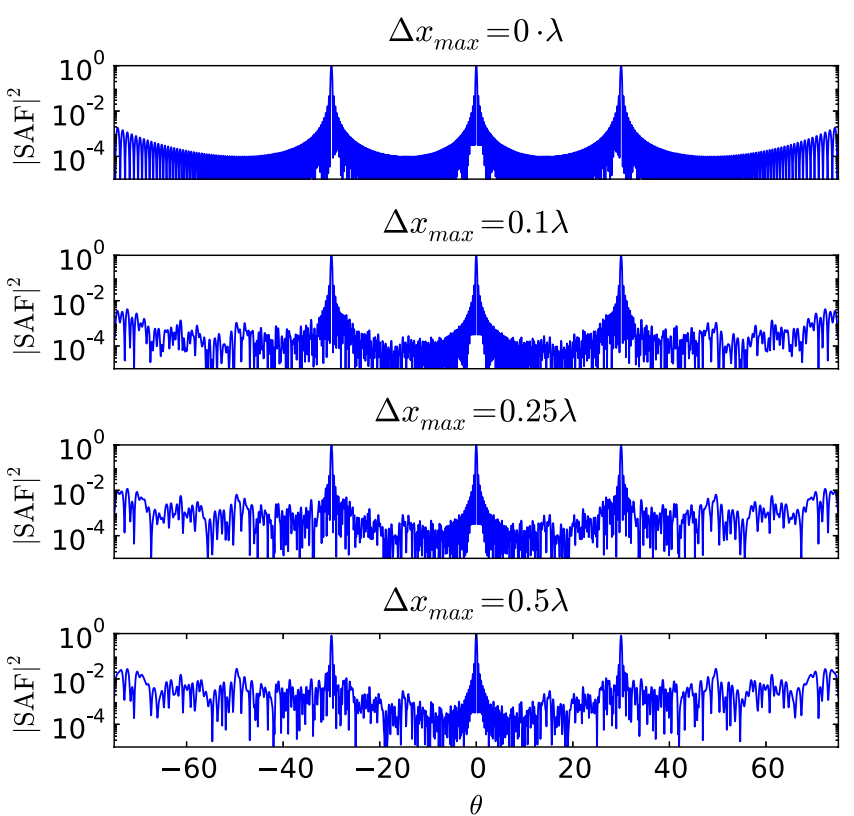

Fig. 9. Results for the same setup as in Fig. 5(a), but with inplane movement as specified in Eq. (20).

By simulating Eq. (21), we get the results shown in Fig. 9. Comparing this with Eq. (21), it can be seen that in-plane translation will never affect the specular mode response (mode 0 ) since $\sin \theta=0$ in that direction, which means that $\operatorname{SAF}(0)=1$ no matter the randomization. For larger angles, the effect will be more and more prominent, though, since $\sin \theta$ is larger and the preferred phase will be less prominent in the phase distribution. This is a huge limitation with respect to creating an effect with no visible diffraction, but could add some randomness to large angles. This is also what is observed in [15].

It should be noted that the displacements in Fig. 9 are quite large, and it would require a structure with lots of air in between as for the structure in this example not to overlap or couple significantly.

\section{Color Representation of SAFs}

To give an idea of the interpretation of the obtained results in terms of color effects for a surface, this section will present some of the SAFs converted to RGB values [26] for given sizes. The conversion used can be found in [27]. The examples used all have a period of $\ell_{x}=2 \mu \mathrm{m}$, and differ by having (1) a random height variation drawn from a uniform distribution between 0 and $110 \mathrm{~nm}$; (2) a random height variation drawn from a uniform distribution between 0 and $220 \mathrm{~nm}$; (3) a random height variation drawn from a uniform distribution between 0 and $1500 \mathrm{~nm}$; (4) and a uniform binary height displacement with the values 0 and $110 \mathrm{~nm}$, which corresponds to the parameters chosen in [14]. The results are presented in Fig. 10.

What can be seen from these plots is that if a uniform random distribution is chosen, and we are designing for a color around a certain wavelength, $\lambda$, then $1 / 2 \lambda$ should be chosen as the upper limit for the
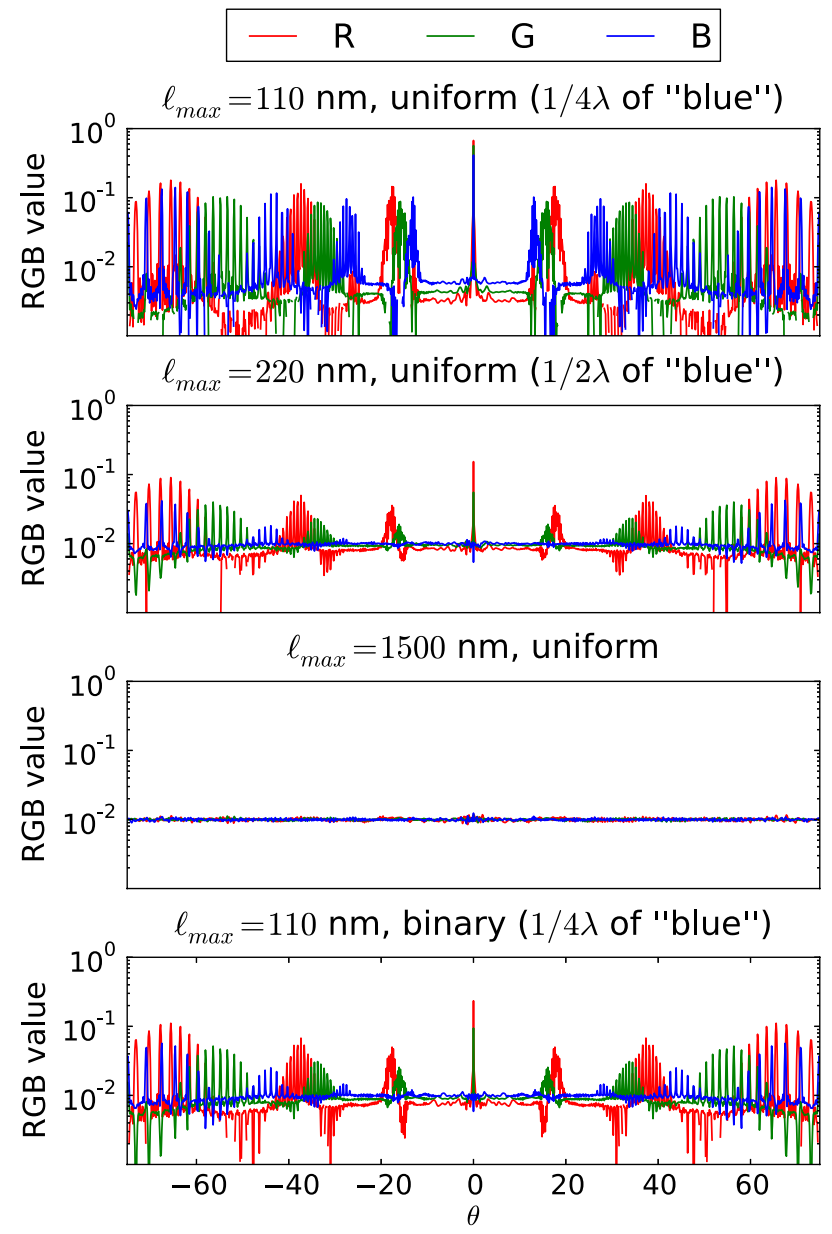

Fig. 10. Color representation of different SAFs. All unit structures are repeated with a period of $2 \mu \mathrm{m}$, and incoherence has been taken into account by averaging over 200 samples. The oscillatory effect seen at large angles is due to the fact that the angular spacing between wavelengths gets larger. Notice how the response for blue is flat for all plots except the uniform random distribution only going to $110 \mathrm{~nm}$.

uniform distribution (blue has a wavelength around $440 \mathrm{~nm}$ ), whereas a binary random distribution will require only $1 / 4 \lambda$, and, as shown earlier, choosing $1 / 2 \lambda$ will give a strong diffraction pattern. Furthermore it is seen that choosing a large upper limit of the uniform distribution makes a good choice for giving a flat distribution of all colors. This could have some practical issues, though, since the translation may be large compared to the unit structure and possibly violate the assumptions stated earlier. All in all, this suggests that when designing random patterns for a given color, it may be beneficial to use binary randomness if possible, since this gives the smallest translation of the structures and in many cases will be easier to produce by, e.g., an etching process as in [14]. Not all colors can be represented by just one relatively small band in the visible range such as, e.g., magenta or white, and if that is not possible other means are needed. This could, for example, be large uniform randomness or possibly the concept of binary randomness but expanded to more levels, 


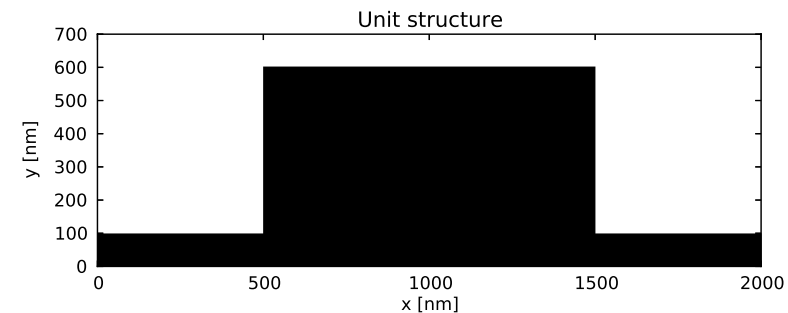

(a)

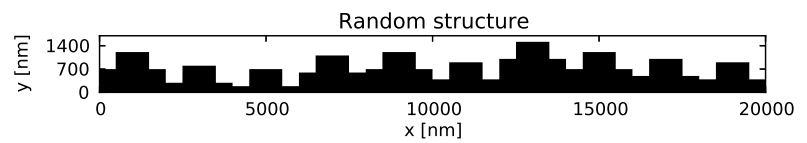

(b)

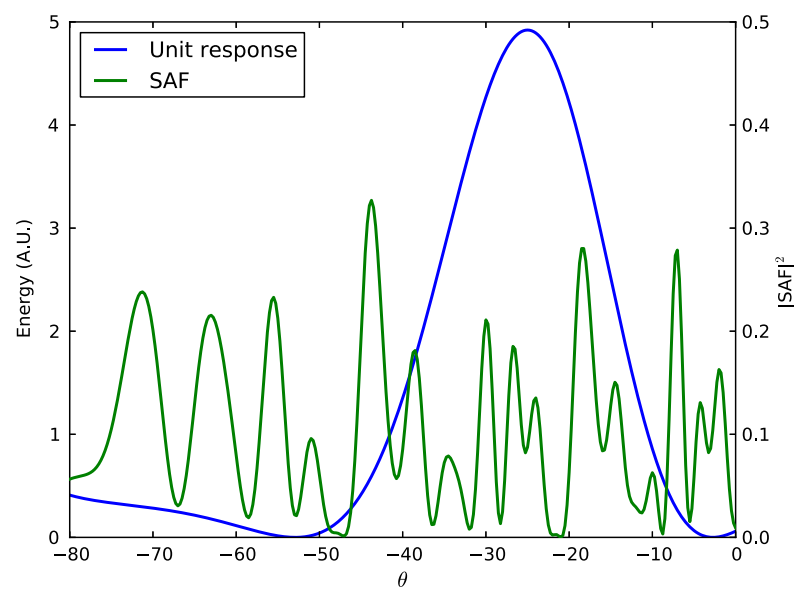

(c)

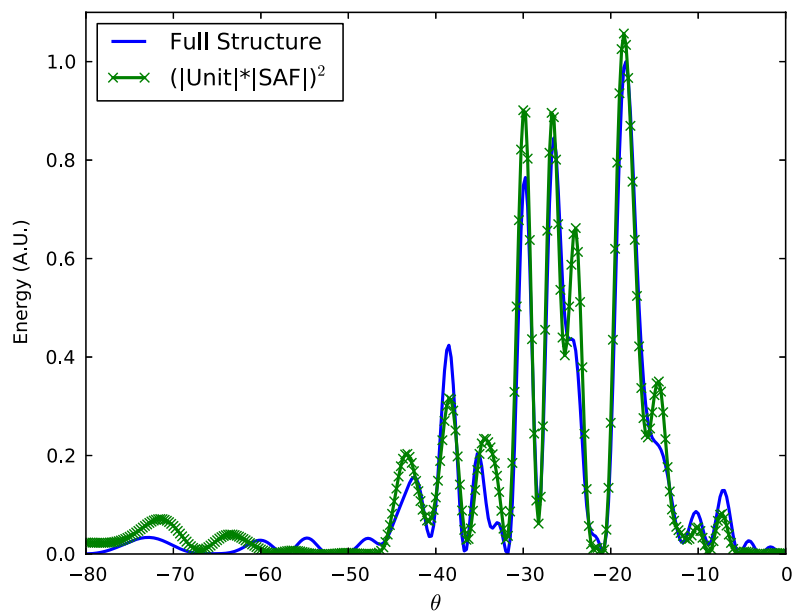

(d)

Fig. 11. Verification of the proposed method by comparison to a full-wave simulation for the $E_{z}$ polarized case (c.f. [26]). (a) Unit structure with black representing material having $\overline{\varepsilon_{r}}=$ 3.5 and white being air. (b) Ten random height translations of this structure with the heights chosen as described in the text. Both structures have periodic boundary conditions. The far-field transformation for (a) and (b) are calculated on the lines $y=700 \mathrm{~nm}$, for $x \in[0,2000] \mathrm{nm}$ and $y=1500 \mathrm{~nm}$, for $x \in[0,20000] \mathrm{nm}$, respectively. (c) Unit response for (a) simulated at $\lambda=750 \mathrm{~nm}$ as well as the SAF calculated from Eq. (22). (d) Comparison of the approximation and full simulation reference. which are chosen such that they provide destructive interference at several wavelengths. The method will be dependent on the target color, and further investigations are therefore left for the concrete cases that a scientist or designer may have.

\section{Conclusion}

A method for analyzing translatorial randomness of repeated structures has been presented, and it has been shown how existing observations in the literature can be explained from this. The results presented are intented to push forward the understanding of these randomization phenomena; for example, it explains why height randomization removes diffraction patterns much better than inplane randomization and how to test the effect of different height distributions. This saves time and gives more insight in the analysis of random phenomena compared to the more expensive full-wave simulation of repeated structures as has been seen earlier in the investigation of random effects. The method also makes it possible to apply a systematic approach for choosing randomization characteristics when designing surfaces with new color effects based on a unit structure.

\section{Appendix A: Verification Example}

To verify the presented method, a unit structure as seen in Fig. 11(a) has been repeated 10 times with a random height translation and lit by a wave having an incident angle of $25^{\circ}$ to the normal of the surface. The response has then been calculated using Eq. (12) and compared to a full-wave simulation of the whole structure [see Fig. 11(b)]. This means that

$$
\hat{\mathbf{k}}=\left(\sin \theta_{i}, 0, \cos \theta_{i}\right), \quad \Delta \mathbf{r}_{n}=\left(n \ell_{x}, 0, \ell_{z, n}\right),
$$

where $\theta_{i}=180^{\circ}+25^{\circ}, \quad \ell_{x}=2 \mu \mathrm{m}$, and $\left\{\ell_{z, n}\right\}=$ $\{0.6,0.2,0.1,0.5,0.6,0.3,0.9,0.6,0.4,0.3\} \mu \mathrm{m}$. The results of the simulation are presented in Fig. 11. By visual inspection, a fairly good match can be found; note in particular how most peak positions are caused purely by the translatorial properties and how the intensity distribution is dominated by the unit structure response.

The author would like to thank Professors Olav Breinbjerg and Ole Sigmund for discussions and excellent feedback regarding this paper. The author is grateful for the support from the Danish National Technology Foundation through the ODAAS project.

\section{References}

1. R. Hooke, Micrographia, http://www.gutenberg.org (1665).

2. A. R. Parker, "515 million years of structural colour," J. Opt. A 2, R15-R28 (2000)

3. P. Vukusic, J. R. Sambles, C. R. Lawrence, and R. J. Wootton, "Quantified interference and diffraction in single Morpho butterfly scales," Proc. R. Soc. B 266, 1403-1411 (1999).

4. N. Okada, D. Zhu, D. Cai, J. B. Cole, M. Kambe, and S. Kinoshita, "Rendering Morpho butterflies based on high accuracy nano-optical simulation," J. Opt. 42, 25-36 (2013).

5. C. W. Mason, "Structural colors in insects. II," J. Phys. Chem., 31, 321-354 (1927). 
6. W. Lippert and K. Gentil, "Über Lamellare Feinstrukturen bei den Schillerschuppen der Schmetterlinge vom Urania- und Morpho-typ Z," Morph. Ökol. Tiere 48, 115-122 (1959).

7. A. R. Parker, T. Lenau, and A. Saito, "Biomimetics of optical nanostructures," in Biomimetics in Photonics (CRC Press, 2012), pp. 55-115.

8. S. Kinoshita, D. Zhu, and A. Saito, "Modeling and simulation of structural colors," in Biomimetics in Photonics (CRC Press, 2012), pp. 191-242.

9. P. Licinio, "Diffraction by disordered gratings and the DebyeWaller effect," Am. J. Phys. 67, 1013-1016 (1999).

10. J. M. Rico-García and L. M. Sanchez-Brea, "Binary gratings with random heights," Appl. Opt. 48, 3062-3069 (2009).

11. T. Buß, J. Teisseire, and S. Mazoyer, "Controlled angular redirection of light via nanoimprinted disordered gratings," Appl. Opt. 52, 709-716 (2013).

12. F. Pratesi, M. Burresi, F. Riboli, K. Vynck, and D. S. Wiersma, "Disordered photonic structures for light harvesting in solar cells," Opt. Express 21, A460-A468 (2013).

13. R. T. Lee and G. S. Smith, "Detailed electromagnetic simulation for the structural color of butterfly wings," Appl. Opt. 48, 4177-4190 (2009).

14. A. Saito, Y. Miyamura, Y. Ishikawa, J. Murase, M. AkaiKasaya, and Y. Kuwahara, "Reproduction, mass-production and control of the Morpho-butterfly's blue," Ad. Fabric. Technol. Micro/Nano Optics and Photonics II 7205, 720506 (2009).

15. A. Saito, M. Yonezawa, J. Murase, S. Juodkazis, V. Mizeikis, M. Akai-Kasaya, and Y. Kuwahara, "Numerical analysis on the optical role of nano-randomness on the Morpho butterfly's scale," J. Nanosci. Nanotech. 11, 2785-2792 (2011).

16. M. A. Steindorfer and V. Schmidt, "Detailed simulation of structural color generation inspired by the Morpho butterfly," Opt. Express 20, 21485-21494 (2012).

17. C. A. Balanis, Advanced Engineering Electromagnetics, 2nd ed. (Wiley, 2012).
18. M. Zhou, S. B. Sørensen, E. Jørgensen, P. Meincke, O. S. Kim, and O. Breinbjerg, "An accurate technique for calculation of radiation from printed reflect arrays," IEEE Antennas and Wireless Propagation Lett. 10, 1081-1084 (2011).

19. D. Zhu, S. Kinoshita, D. Cai, and J. Cole, "Investigation of structural colors in Morpho butterflies using the nonstandard-finite-difference time-domain method: effects of alternately stacked shelves and ridge density," Phys. Rev. E 80, 051924 (2009).

20. P. Dutré, K. Bala, and P. Bekaert, Advanced Global Illumination (A K Peters, 2006).

21. J. E. Harvey, C. L. Vernold, A. Krywonos, and P. L. Thompson, "Diffracted radiance: a fundamental quantity in nonparaxial scalar diffraction theory," Appl. Opt. 38, 6469-6481 (1999).

22. T. Antonakakis, F. Bada, A. Belkhir, K. Cherednichenko, S. Cooper, R. Craster, G. Demesy, J. DeSanto, G. Granet, B. Gralak, S. Guenneau, D. Maystre, A. Nicolet, B. Stout, F. Zolla, and B. Vial, Gratings: Theory and Numeric Applications, 1st ed. (Presses Eniversitaires de Provence, 2012).

23. R. N. Bracewell, The Fourier Transform and its Applications, 3rd ed. (McGraw Hill, 2000).

24. S. Kinoshita, S. Yoshioka, Y. Fujii, and N. Okamoto, "Photophysics of structural color in the Morpho butterflies," Forma, 17, 103-121 (2002).

25. R. T. Lee, "A novel method for incorporating periodic boundaries into the FDTD method and the application to the study of structural color of insects," Ph.D. thesis (Georgia Institute of Technology, 2009).

26. R. S. Berns, F. W. Billmeyer, and M. Saltzman, Billmeyer and Saltzman's Principles of Color Technology (Wiley-Interscience Publication, 2000).

27. J. Andkjær, V. E. Johansen, K. S. Friis, and O. Sigmund, "Inverse design of nanostructured surfaces for color effects," J. Opt. Soc. Am. B 31, 164-174 (2014). 